\title{
A REALISTIC INTERPRETATION OF LATTICE GAUGE THEORIES
}

\author{
Miguel Lorente \\ Departamento de Física, Facultad de Ciencias \\ Universidad de Oviedo \\ E-3300\%, Oviedo, Spain
}

Following recent assumptions to unify quantum mechanics and general relativity, the structure of spacetime is suppose to be a consequence of the relations among some fundamental objects, and its concept can be formulated without the reference to the intuition. As physical consequences the continuous laws should be translated in to difference equations and the lattice field theories should be interpreted as a realistic model.

Key words: spacetime, real lattice, process, relations.

\section{RECENT ASSUMPTIONS TO UNIFY QUANTUM ME- CHANICS AND THE STRUCTURE OF SPACETIME}

One of the most difficult problems to unify the postulates of QM and general relativity is the different conceptions of spacetime. In QM the spacetime is a container where the fields are distinguished by their position and interactions, in the theory of relativity the gravitational field is identified with the metrical properties of spacetime. Recently several authors have tried to overcome this difficulty by deriving the structure of spacetime from the properties of fundamental processes described by QM.

According to Joseph M. Jauch, the set of propositions of a physical system in QM replaces the phase space [1]. In classical mechanics the underlying spacetime is necessary to impose the initial conditions that determined the solution of equations of motion. In QM the equation of motions are substituted by the set of propositions based on the superposition of the simplest yes-no experiments. The axiomatic form of this structure gives rise to the calculus of propositions, that do not presuppose the space time. The physical state is the result of a series of physical manipulations on the set of propositions. 
Karl F. von Weizsaecker gives a more explicit connections between quantum theory and the concept of time and space [2]. All the quantum processes can be reduced to binary alternatives (equivalent to yes-no experiments). The interaction among these fundamental entities, which he calls "urs", gives rise to physical system and the structure of spacetime is the set of relations among the binary alternatives.

There are two important postulates: (i) the number of actual alternatives that determine a physical is finite, because they represent real properties; (ii) the number of possible alternatives is infinite due to the indeterministic nature of the quantum processes. As a consequence, the description of facts is given by discrete variables, but the physical laws are given in terms of continuous functions.

Roger Penrose does not pressupose an underlying spacetime for the physical processes [3]. The starting point is the total angular momentum of some fundamental units, the interactions of which produce a discrete network. "My model, says Penrose, works with objects and interactions between objects. An object is thus located either directionally or positionally in terms of its relations with other objects. One does not really need a space to begin with. The notion of space comes out as a convenience at the end."

According to David Finkelstein the world is a network of quantum processes, which he calls "monads"[4]. Every process in nature is a finite assembly of elementary processes, namely, of creation and destruction, and the structure of Spacetime is the set of all elementary processes and their relations.

\section{EPISTEMOLOGY OF THESE MODELS}

In order to understand better these models it would be useful to consider three levels of human knowledge in the comprehension of the physical world[5]:

Level 1: Physical magnitudes, such as distances, intervals, force, mass, charge, that are given by our sensation and perceptions.

Level 2: Mathematical structures, that are the result of metrical properties given by measurements and numerical relations among them.

Level 3: Fundamental concepts, representing the ontological properties of physical world given by our intelligence in an attempt to know the reality. This level of knowledge is not accepted by some philosophical positions like logical positivismus, conventionalismus, neokantismus.

There must be some connections between the three levels. In QM the theoretical models of microphysics in level 2 are related to observable mag- 
nitudes in level 1 by correspondence laws. If we accept level 3 it should be connected to level 2 and to level 1 (through level 2). In fact, the rules governing the constructions of theoretical models in level 2 must be grounded in some fundamental (ontological) properties of the physical world.

We can now raise the following question: in theoretical models of level 2 there are primitive and derived concepts, the last ones are obtained from the first ones by mathematical formulas. Are space and time primitive or derived concepts? If the second answer is given the description of the world in level 2 should not include as primitives the geometrical objects such as lines, planes, surfaces.

\section{MODERN THEORIES ON THE STRUCTURE OF SPACE- TIME}

In order to answer the last question it is convenient to recall the different interpretations of the concepts of space and time [6]. They are usually divided in three classes.

(a) Dualistic theories: Space is a container where the particles and waves are moving. Time is also a separated entity with respect to which the motion takes place. Therefore space and time are absolute and can be thinked of in the absence of particles (Newton).

(b) Monistic theories: Spacetime is identified with some properties of matter and can not be concevible without the existence of the later. The field of forces and also the sources are nothing more that geometrical deformations of the Spacetime (Einstein, Kaluza-Klein, Wheeler).

(c) Relational theories: Spacetime consists of the set of relations among some fundamental objects: monads (Leibniz), units (Penrose), processes (Weisaecker, Finkelstein), preparticles (Bunge, García Sucre), objects (Hilbert).

In Sec. 1 we have mentioned some of these authors. We expand in some detail Leibniz's and Hilbert's conception. According to Leibniz [7] "time is the order of points (monads) non existing simultaneously and one is the ratio of the other. Space is the order of points that exist simultaneously and are connected by mutual interactions. Space is nothing more that the set of all points and their relations. One point is here if it has relations with some particular points around it. A point changes its position if it changes its relations from some points to different ones. Motion is the change of different positions in time".

In his Foundation of Geometry, Hilbert has proposed an axiomatic approach to Euclidean geometry[8], according to which the concept of space 
is constructed with the help of some logical properties. He distinguishes two types of axiomatization: i) material, by which the concept of space is taken from observation and intuition and ii) formal, in which the concept of space is derived from some formal properties of axioms and inferences without the recourse to the intuition or the observation (his famous expression, "We could say always instead of points, lines and planes, chairs, tables and glasses of beer," confirms his position in favor of the formal axiomatization) The concepts of point, straight line, and plane can be reduced to pure logical relations.

\section{A RELATIONAL THEORY OF SPACETIME}

Following the assumption of the last section now we give an explicit construction of a formal structure of Spacetime, without the recourse to intuition. We can think of a set of fundamental objects acting among themselves, giving rise to a network of relations. These relations do not pressupose some space. The objects are nowhere if we consider them as elements of the physical world in level 2. In order to be specific we take as a naive network a three-dimensional cubic lattice. Obviously the network can be taken with different structure, such as, triangular, quasiperiodic or random lattices. In order to make connection with the euclidean geometry we take, for simplicity, a infinite set of interacting points in the relation 1 to 4 . The set of all relations form a two-dimensional lattice, in which we can define:

A path is the connection between two different points, say, A and B, through points that are pairwise neighbours.

The length of a path is the numbers of points contained in the path, including the first and the last one.

A minimal path is a path with minimal length (in the picture the two paths between $\mathrm{A}$ and $\mathrm{B}$ are minimal). Between two point there can be different minimal paths.

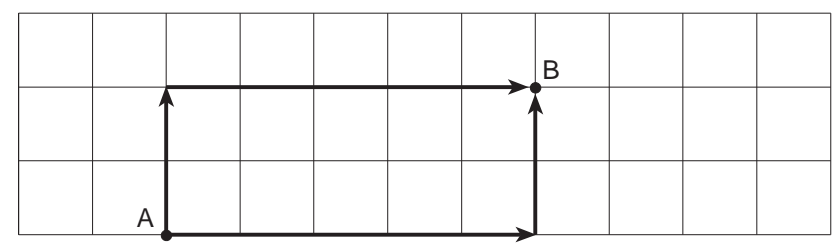

A principal straight line is a indefinite set of points in the lattice, such that each of them is contiguous to other two, and the minimal path between two arbitrary points of this line is always unique. 
Theorem 1. Through a point of a 2-dimensional square lattice pass only two different principal straight lines (they are called orthogonal straight lines).

Theorem 2. Two principal straight lines that are not orthogonal have all the points either in common or separated (in the last case they are called paralell straight lines).

From these two theorem we can define Cartesian (discrete) coordinates and an Euclidean space where the postulates of Hilbert can be applied (with the exception of the axioms of continuity). This structure of 2-dimensional space can be easily generalized to 3-dimensional cubic lattice. As we mentioned, those assumptions for the structure of space are given in level 2 , but it corresponds to the properties of physical space described in level 1 by our sensations.

In order to introduce the relation that correspond to time we start with only two fundamental objects acting among themselves:

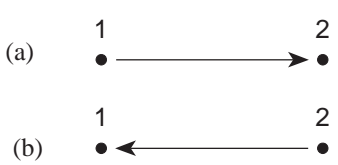

In (a), 1 is acting on 2, and in (b) 2 is acting on 1 . But the action of 1 on 2 is supposed to be a necessary condition for the action of 2 on 1 , and similarly the action of 2 on 1 is supposed to be a necessary condition for a new action of 1 on 2 . Thus we can think of a chain of mutual interactions arranged in a series of necessary conditions. This picture has to be enlarged for the whole lattice. We take a set of interacting objects in the relations 1 to 2 .
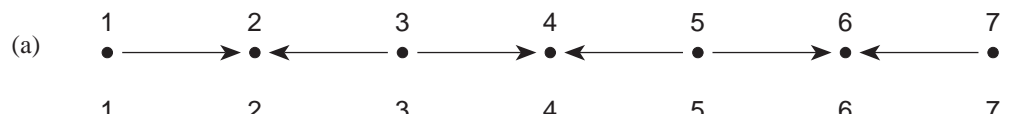

(b)

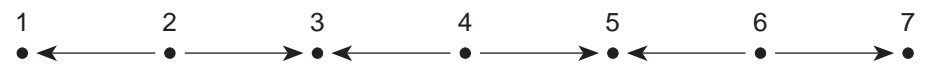

In (a), 1 is acting on 2, 3 is acting on 2 and 4, 5 is acting on 4 and 6 , 7 is acting on 6 . In (b), 2 is acting or 1 and 3, 4 is acting on 3 and 5,6 is acting on 5 an 7 . 
We postulate that the actions of (a) are necessary conditions for the actions of (b) and the actions of (b) are necessary conditions for a further action of type (a) an so on.

Now take a network of objects acting in the relation 1 to 4 .

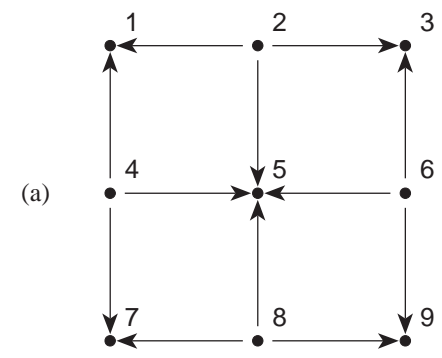

(b)

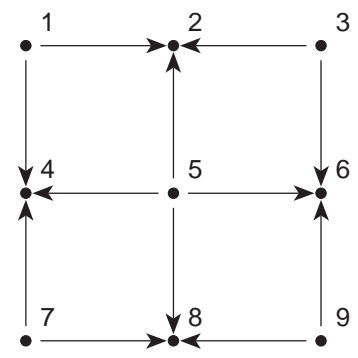

In (a), 2 is acting on $1,3,5 ; 4$ is acting on $1,5,7 ; 6$ is acting on 3,5 , $9 ; 8$ is acting on 5,7,9. In (b), 1 is acting on 2 and $4 ; 3$ is acting on 2 and 6 ; 5 is acting on 2, 4, 6, 8; 7 is acting on 4 and $8 ; 9$ is acting on 6 and 8 . As before we postulate that the actions of (a) be necessary conditions for the actions of (b) and so on. These logical properties of interactions belong to level 2 and do not pressupose the concept of time, but they can be put in correspondence with the physical properties of time given in level 1 .

In level 3 there must be some ontological properties corresponding to the objects and interactions described in level 2.

In our model the most essential character of material entities is not the extension but their capacity to produce effects in other object (external causality). There is a causal relation between cause and effect and the logical necessity that was introduced in the last paragragh for the interpretation of time has its ontological ground in the principle of causality by wich the effect cannot be produced without its cause.

\section{PHYSICAL CONSEQUENCES OF THE MODEL}

The assumption of relational theory of Spacetime with a particular structure of cubic lattice, implies some physical consequences for the classical as well for the quantum physics:

(i) The Spacetime is discrete, therefore the physical laws are written in the language of finite differences. The solutions have to be described by continuous function of discrete variables [10].

(ii) Lattice gauge theories are not only a mathematical tool but a realistic theory, because they correspond to the underlying discrete structure 
of Spacetime. Some correspondence law must be given to make connection with the experimental world [11].

(iii) The symmetry of the model is still Poincaré transformation, although one has to select those integral transformations that keep the lattice invariant [12].

(iv) Some experimental test.Although there are infinite number of integral Lorentz transformations, and the continuous Lorentz transformations is a dense set, there are only 24 pure rotations that keep the lattice invariant. Therefore there is a broken SO (3) symmetry that leads to non-isotropy of the world. This means that one could fine in principle some preferred direction either in the microphysical world or in the large scale of the universe. An other physical application of the model could be detected in the discrete mass spectrum as a natural consequence of the elementary time interval an estimation of wich by actual calculations gives about $10^{-36}$ sec.

In order to prove this we summarized some mathematical results of lattice field theories [13].

We introduce the method of finite differences for the Klein-Gordon scalar field. An explicit scheme for the wave equation consistent with the continuous case (the truncation error is of second orden with respect to space and time variables) can be constructed as follows:

$$
\left(\frac{1}{\tau^{2}} \nabla_{n} \Delta_{n} \tilde{\nabla}_{j} \tilde{\Delta}_{j}-\frac{1}{\varepsilon^{2}} \nabla_{j} \Delta_{j} \tilde{\nabla}_{n} \tilde{\Delta}_{n}+M^{2} \tilde{\nabla}_{n} \tilde{\Delta}_{n} \tilde{\nabla}_{j} \tilde{\Delta}_{j}\right) \phi_{j}^{n}=0 ;
$$

here in the field is defined in the grid points of the $(1+1)$-dimensional lattice $\phi_{j}^{n} \equiv \phi(j \varepsilon, n \tau), \varepsilon, \tau$ being the space and time fundamental intervals, $j, n$ integer numbers, and $\Delta_{j}\left(\nabla_{j}\right)$ are the forward (backward) differences with respect to the space index, $\tilde{\Delta}_{j}\left(\tilde{\nabla}_{j}\right)$ the forward (backward) averages, and similarly for the time index.

Using the method of separation of variables, it can easily be proved that the following functions of discrete variables are solutions of the wave equation (11):

$$
f_{j}^{n}(k, \omega)=\left(\frac{1+\frac{1}{2} i \varepsilon k}{1-\frac{1}{2} i \varepsilon k}\right)^{j}\left(\frac{1-\frac{1}{2} i \tau \omega}{1+\frac{1}{2} i \tau \omega}\right)^{n},
$$

provided the "dispersion relation" is satisfied:

$$
\omega^{2}-k^{2}=M^{2}
$$

$\mathrm{M}$, being the mass of the particle. 
In the limit, $j \rightarrow \infty, \quad n \rightarrow \infty, \quad j \varepsilon \rightarrow x, \quad n \tau \rightarrow t$, the functions (2) become plane wave solutions

$$
f_{j}^{n}(k, \omega) \rightarrow \exp i(k x-\omega t) .
$$

Imposing boundary conditions on the space indices,

$$
f_{o}^{n}(k, \omega)=f_{N}^{n}(k, \omega)
$$

we get

$$
k_{m}=\frac{2}{\varepsilon} \tan \frac{\pi m}{N}, \quad m=0,1, \ldots, N-1
$$

therefore

$$
\omega= \pm\left(k_{m}^{2}+M^{2}\right)^{1 / 2} .
$$

For the positive energy solutions we define

$$
\omega_{m}=+\left(k_{m}^{2}+M^{2}\right)^{1 / 2} .
$$

Starting from the wave equation (11), we can construct a current vector. Multiplying (11) by $\tilde{\nabla}_{n} \tilde{\Delta}_{n} \tilde{\nabla}_{j} \tilde{\Delta}_{j} \phi_{j}^{* n}$ from the left, and multiplying the complex conjugate of the wave equation by $\tilde{\nabla}_{n} \tilde{\Delta}_{n} \tilde{\nabla}_{j} \tilde{\Delta}_{j} \phi_{j}^{n}$ from the right, substracting both results, we obtain the "conservation law"

$$
\frac{1}{\varepsilon} \nabla_{j} j_{1}-i \frac{1}{\tau} \nabla_{n} j_{4}=0
$$

where

$$
\begin{aligned}
j_{1} \equiv & i\left[\frac{1}{\varepsilon} \Delta_{j}\left(\tilde{\nabla}_{n} \tilde{\Delta}_{n} \phi_{j}^{* n}\right) \tilde{\Delta}_{j}\left(\tilde{\nabla}_{n} \tilde{\Delta}_{n} \phi_{j}^{n}\right)\right. \\
& \left.-\tilde{\Delta}_{j}\left(\tilde{\nabla}_{n} \tilde{\Delta}_{n} \phi_{j}^{* n}\right) \frac{1}{\varepsilon} \Delta_{j}\left(\tilde{\nabla}_{n} \tilde{\Delta}_{n} \phi_{j}^{n}\right)\right], \\
j_{4} \equiv & i \rho \equiv\left[\frac{1}{\tau} \Delta_{n}\left(\tilde{\nabla}_{j} \tilde{\Delta}_{j} \phi_{j}^{* n}\right) \tilde{\Delta}_{n}\left(\tilde{\nabla}_{j} \tilde{\Delta}_{j} \phi_{j}^{n}\right)\right. \\
& \left.-\tilde{\Delta}_{n}\left(\tilde{\nabla}_{j} \tilde{\Delta}_{j} \phi_{j}^{* n}\right) \frac{1}{\tau} \Delta_{n}\left(\tilde{\nabla}_{j} \tilde{\Delta}_{j} \phi_{j}^{n}\right)\right]
\end{aligned}
$$

are the spatial and time component, respectively, of the charge vector current on the lattice.

The charge density $\rho$ suggest that we can substitute the scalar field $\phi(x, t)$ by the smeared field $\tilde{\nabla}_{j} \tilde{\Delta}_{j} \phi_{j}^{n}$ and $\phi^{*}(x, t)$ by $\tilde{\nabla}_{j} \tilde{\Delta}_{j} \phi_{j}^{* n}$. 
A suitable Hamiltonian for the real field $\phi_{j}^{n}$ and its conjugate momentum $\pi_{j}^{n}$ can be defined as follows:

$$
\begin{aligned}
H_{n}= & \varepsilon \sum_{j=0}^{N-1} \frac{1}{2}\left\{\left(\tilde{\nabla}_{j} \tilde{\Delta}_{j} \pi_{j}^{n}\right)^{2}-\frac{1}{\varepsilon^{2}}\left(\nabla_{j} \Delta_{j} \phi_{j}^{n}\right)\left(\tilde{\nabla}_{j} \tilde{\Delta}_{j} \phi_{j}^{n}\right)\right. \\
& \left.+M^{2}\left(\tilde{\nabla}_{j} \tilde{\Delta}_{j} \phi_{j}^{n}\right)^{2}\right\} \equiv \varepsilon \sum_{j=0}^{N-1} \mathcal{H}_{j}^{n} .
\end{aligned}
$$

As in the continuous case, we can derived the Hamilton equations of motions, varying the Hamiltonian density $\mathcal{H}_{j}^{n}$ first with respect to the promediate momentum and secondly with respect to scalar field:

$$
\begin{aligned}
\frac{1}{\tau} \Delta_{n}\left(\tilde{\nabla}_{j} \tilde{\Delta}_{j} \phi_{j}^{n}\right) & =\frac{\partial \mathcal{H}_{j}^{n}}{\partial\left(\tilde{\nabla}_{j} \tilde{\Delta}_{j} \pi_{j}^{n}\right)}=\tilde{\Delta}_{n}\left(\tilde{\nabla}_{j} \tilde{\Delta}_{j} \pi_{j}^{n}\right) \\
\frac{1}{\tau} \Delta_{n}\left(\tilde{\nabla}_{j} \tilde{\Delta}_{j} \pi_{j}^{n}\right) & =-\frac{\partial \mathcal{H}_{j}^{n}}{\partial\left(\tilde{\nabla}_{j} \tilde{\Delta}_{j} \phi_{j}^{n}\right)} \\
& =\tilde{\Delta}_{n}\left(\frac{1}{\varepsilon^{2}} \nabla_{j} \Delta_{j} \phi_{j}^{n}-M^{2} \tilde{\nabla}_{j} \tilde{\Delta}_{j} \phi_{j}^{n}\right) .
\end{aligned}
$$

Applying the difference operator $\frac{1}{\tau} \nabla_{n}$ on both sides of (13) and substituting (14) in the result, we recover the wave equation (11).

Using (13) and (14), it can easily be proved that the Hamiltonian (12) is independent of the time index $n$, namely:

$$
\nabla_{n} H_{n}=\Delta_{n} H_{n}=0 \text {. }
$$

Since the plane wave solutions $f_{j}^{n}\left(k_{m}, \omega_{m}\right)(m=0,1, \ldots, N-1)$ form a complete set of orthogonal functions, we can expand the smeared field and its conjugate momentum as

$$
\begin{aligned}
& \tilde{\nabla}_{j} \tilde{\Delta}_{j} \phi_{j}^{n}=\frac{1}{\sqrt{N \varepsilon}} \sum_{m=-N / 2}^{N / 2-1} \frac{1}{\sqrt{2 \omega_{m}}}\left(a_{m} f_{j}^{n}\left(k_{m}, \omega_{m}\right)+a_{m}^{*} f_{j}^{* n}\left(k_{m}, \omega_{m}\right)\right), \\
& \tilde{\nabla}_{j} \tilde{\Delta}_{j} \pi_{j}^{n}=\frac{-i}{\sqrt{N \varepsilon}} \sum_{m=-N / 2}^{N / 2-1} \sqrt{\frac{\omega_{m}}{2}}\left(a_{m} f_{j}^{n}\left(k_{m}, \omega_{m}\right)-a_{m}^{*} f_{j}^{* n}\left(k_{m}, \omega_{m}\right)\right) .
\end{aligned}
$$

In order to make connection of our scheme with the Einstein-de Broglie relations $E=\hbar \omega, \quad p=\hbar k$, we take, for the period $T$ and wavelength $\lambda$ of the discrete plane waves functions (2) and (5),

$$
T=N \tau, \quad \lambda=N \varepsilon
$$


and, for the phase velocity,

$$
v_{p}=\frac{\lambda}{T}=\frac{\varepsilon}{\tau}
$$

We have defined the wave number and the angular frequency of the wave functions as:

$$
k_{m}=\frac{2}{\varepsilon} \tan \frac{\pi m}{N}, \quad \omega_{m}=\frac{2}{\tau} \tan \frac{\pi m}{N}, \quad m=0,1, \ldots, N-1 .
$$

Substituting the Einstein-de Broglie relations in the relativistic expresion $E^{2}-p^{2}=M^{2}$ (we use natural units $\hbar=c=1$ ), we obtain

$$
\omega_{m}^{2}-k_{m}^{2}=\omega_{m}^{2}\left(1-\frac{\tau^{2}}{\varepsilon^{2}}\right)=\omega_{m}^{2}\left(1-\frac{1}{v_{p}^{2}}\right)=M^{2} .
$$

Since the phase velocity and group velocity satisfy $v_{p} v_{g}=1$, we have finally

$$
\omega_{m}^{2}=\frac{M^{2}}{1-v_{g}^{2}},
$$

hence $M$ has $m$-dependent discrete spectrum.

\section{References}

[1] J. Jauch, Foundations of Quantum Mechanics (Addison-Wesley, Reading,1968).

[2] C.F. Weizsaecker, "Reconstruction of quantum mechanics," in Quantum Theory and the Structure of Time and Space, L. Castell and C. Weizsaecker, eds. (Hanser, Munich, 1986).

[3] R. Penrose, "Angular momentum: an approach to combinatorial analysis" in Quantum Theory and Beyond, T. Bastin, ed. (Cambridge University Press, Cambridge, 1971).

[4] D. Finkelstein, "Spacetime code," Phys. Rev. 184 (1968) 1261.

[5] M. Lorente, "A causal interpretation of the structure of space and time," Foundations of Physics, P. Weingartner and G. Dorn, eds. (Hölder,Pichler \& Tempsky, Viena 1986).

[6] M. Lorente, "Modernas Teorías sobre la estructura del espacio-tiempo," Reunión Matemática en honor de A. Dou (Universidad Complutense de Madrid, 1989). 
[7] M. Jammer, Concepts of Space (Cambridge University Press, Cambridge, 1969). According to this author, Leibniz's Monadology was inspired by Maimonides, who, in his Guide for the Perplexed, chap. 73, describes the theory of a discrete space and time.

[8] D. Hilbert, Grundlage der Geometrie (Teubner, Leipzig, 1899). Spanish translation: Fundamentos de la Geometría (C.S.I.C., Madrid, 1991).

[9] See Ref. 5.

[10] M. Lorente, Int. J. Theor. Phys. 4 (1974) 213; 12 (1976) 927; 25 (1986) 55.

[11] M. Lorente, J. Group Theory in Phys.1 (1993) 105.

[12] M. Lorente, "A relativistic invariant scheme for the Klein-Gordon and Dirac fields on the lattice," XIX Int. Coll. on Group Theor. Meth. in Phys. (Editorial Ciemat, Madrid, 1992), p. 395-398.

[13] M. Lorente, "Representations of the classical groups on the lattice" in Symmetries in Science VI, B. Gruber, ed. (Plenum, New York, 1993), pp. 437-454. 